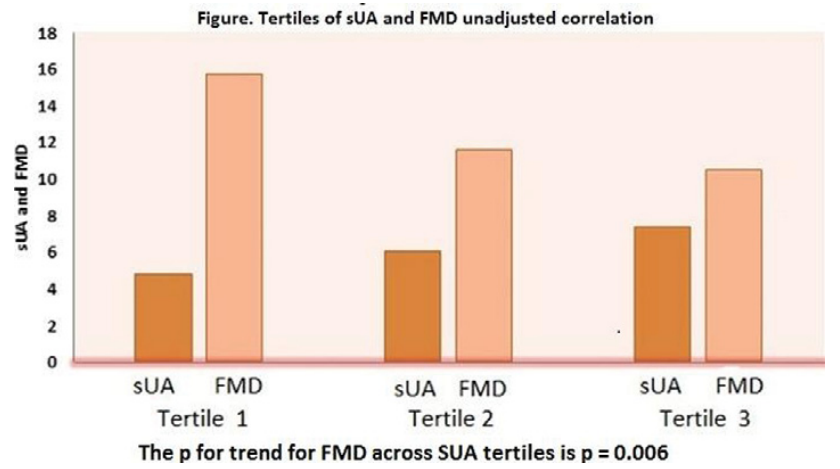

dysfunction or BP. Endothelial dysfunction or BP might be associated with changes in SUA when measured longitudinally in individuals, but not when measured cross-sectionally in populations. Larger studies will be needed to confirm these results.

References:

[1] Ruggiero, C. Am J Cardiol, 2007. 100(1): p. 115-21.

[2] Rossi, R. J Am Coll Cardiol, 2004. 44(8): p. 1636-40.

[3] Feig, D. JAMA, 2008. 300(8): p. 924-32.

Acknowledgements: National Institute of Arthritis and Musculoskeletal and Skin Diseases P50AR060772, K24AR052361 (to KGS).

Disclosure of Interest: M. Saddekni: None declared, K. Saag Grant/research support from: AstraZeneca, Crealta, Takeda, Consultant for: Ardea/AstraZeneca,

Crealta, Takeda, T. Dudenbostel: None declared, D. Calhoun: None declared, S. Oparil Grant/research support from: NHLBI Brigham and Women's Hospital, Center CVD Prev, Novartis Pharmaceutical Corporation, AstraZeneca AB (Duke University), Actelion Pharmaceuticals US,NIH/NHLBI, Merck and Co., Consultant for: Amgen, Bayer, Boehringer Ingelheim, AstraZeneca, Medtronic, GlaxoSmithKline, Forest Labs Inc., D. Feig: None declared, P. Muntner: None declared, P. Foster: None declared, S. Biggers: None declared, E. Rahn: None declared, P. Li: None declared, D. Redden: None declared, A. Gaffo Grant/research support from: Amgen, AstraZeneca, Consultant for: Cymabay, Ardea, Employee of: US Government

DOI: 10.1136/annrheumdis-2017-eular.5313

\section{THU0414 INCIDENCE AND PREDICTORS FOR NEPHROLITHIASIS IN GOUT PATIENTS AND THE GENERAL POPULATION}

A.J. Landgren ${ }^{1}$, L. Jacobsson ${ }^{1}$, U. Lindström ${ }^{1}$, T.Z. Sandström ${ }^{1}$, E. Fjellstedt ${ }^{2}$, P. Drivelegka ${ }^{1}$, V. Sigurdardottir ${ }^{1}$, L. Björkman ${ }^{1}$, M. Dehlin ${ }^{1}$. ${ }^{1}$ Department of Rheumatology and Inflammation Research, Institution of Medicine, Sahlgrenska Academy, University of Gothenburg, Gothenburg; ${ }^{2}$ Department of Nephrology and Transplantation, SUS University Hospital, Malmö, Sweden

Background: A well-known complication of gout is an increased risk for nephrolithiasis (NL). The incidence rate of $\mathrm{NL}$ in the general population varies in different studies between 85 and 170/100 000 person-years, with a peak incidence in the ages 40-49 years. Several medications used in gout patients could affect the risk for NL, including allopurinol, losartan, thiazide- and loop-diuretics. Effect of these medications on risk of NL in gout patients, and the general population, has only scarcely been studied.

Objectives: In this cohortstudy we investigated: 1) overall incidence of NL in gout and general population (GP) controls 2) risk for first time NL in gout patients vs general population (GP) controls, and 3) predictors for first time NL in both groups separately.

Methods: Gout patients were identified from the regional health care database in western Sweden (VEGA), containing ICD10-codes for all regional Healthcare visits from 2000. Matched (birthyear, sex, county) GP controls were selected from the population register. National registers and VEGA were used to retrieve information on comorbidities, socioeconomic factors and current medications at start of follow-up. The study population had to be above 19 years of age, without NL prior to start of follow-up, and living in the Western Swedish Health Care Region (WSHCR). Follow-up began 2006-01-01, or at the first gout-diagnosis if this occurred later, and ended at death, emigration or 2012-12-31, whichever occurred first. Incidence rates (IR) per 1000 person-years and hazardratios (HR) were calculated. Possible predictors for NL were based on risk factors presented in the literature.

Results: 29,968 gout patients and 138,678 matched GP controls were included. In gout patients there were $678 \mathrm{NL}$-events (IR: 6.2 per 1000 pyrs at risk $(95 \% \mathrm{Cl}$ : 5.7-6.6)) and in GP controls 2125 (IR 3.9 per 1000 pyrs at risk (95\% Cl 3.7-4.0)). Risk for NL was increased in gout ( $\mathrm{HR}=1.49,95 \% \mathrm{Cl}$ : $1.35-1.64)$, and was higher in men compared to women $(P<0.0001)$ in all age groups for gout cases and controls. All comorbidities and medications were more frequent in cases compared to controls $(p<0.0001)$ at start of follow-up. Risk-factors for NL such as kidney disease $(K D)$, obesity, diabetes were $2-4$ times more common in gout patients compared to GP controls. Predictor point estimates for NL were similar in gout cases and GP controls (Figure 1), except for losartan which increased the

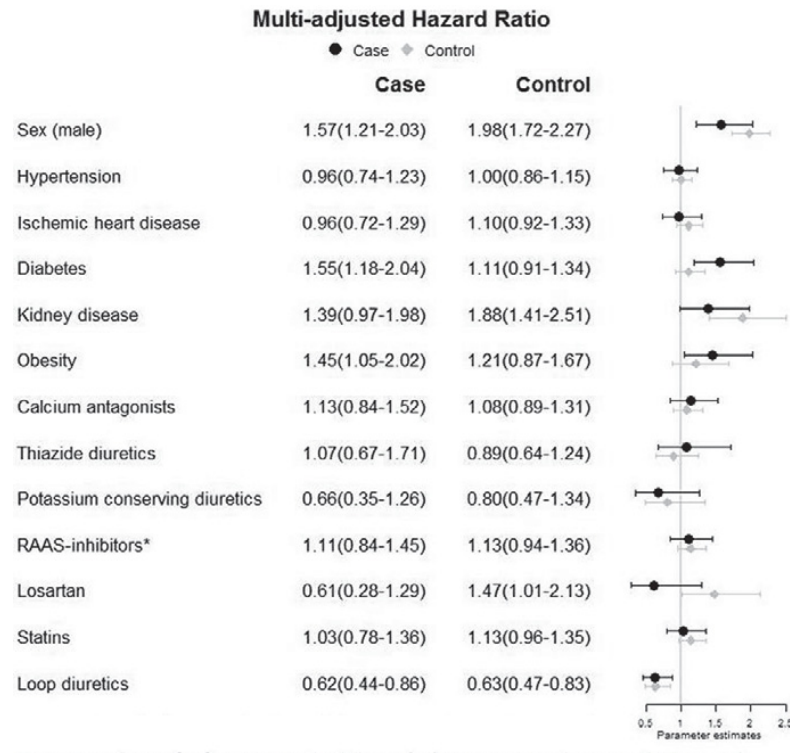

Figure 1 Predictors for first time nephrolithiasis (NL) in patients with gout and GP controls without gout, analyzed by multivariate proportional hazards analyses, adjusting for age and other covoriates in the figure, *Renin-angiotensin-aldosterone-System-Inhibitors excluding losortan

risk of NL in GP controls ( $\mathrm{HR}=1,47,95 \% \mathrm{Cl}: 1.01-2.13)$ but not in gout patients. Loop-diuretics appeared to decrease the risk for NL in both cohorts $(P<0.0001)$, whereas other cardiovascular (CVD) drugs had no effect.

Conclusions: The risk for NL was increased by $50 \%$ in gout patients, compared to controls. Overall pattern of predictors was similar in gout patients and population controls. In patients with gout, male sex, diabetes mellitus (DM), obesity predicted $\mathrm{NL}$, whereas use of loop diuretics was protective. Overall, the most commonly used CVD drugs did not increase the risk for NL in patients with gout.

Disclosure of Interest: None declared

DOI: 10.1136/annrheumdis-2017-eular.2836

\section{THU0415 A STRUCTURED MONITORING PROGRAM FOR DRUG ALLERGY IN PATIENTS NEWLY INITIATED ON ALLOPURINOL}

B.Y. Thong ${ }^{1}$, K.-Y. Gan ${ }^{2}$, B.-H. Ang ${ }^{2} .{ }^{1}$ Rheumatology, Allergy and Immunology; ${ }^{2}$ Pharmacy, Tan Tock Seng Hospital, Singapore, Singapore

Background: Allopurinol allergy (drug eruption, severe cutaneous adverse reactions [SCAR] and drug induced hypersensitivity syndrome [DiHS]) develops during the first 2-12 weeks after initiation. SCAR risk factors include Chinese ethnicity, HLA-B*5801 positivity and chronic kidney disease. HLA-B*5801 testing to prevent SCAR has not been shown to be cost-effective in Singapore.

Objectives: To retrospectively study whether a structured monitoring program (SMP) can lead to early diagnosis of allopurinol allergy and prevent development of SCAR/DiHS.

Methods: SMP patients (cases) managed by rheumatologists were compared with controls managed by non-rheumatologists during the study period 1 Jan 2015 to 30 Jun 2016. Cases upon initiation of allopurinol had baseline full blood count (FBC), serum creatinine ( $\mathrm{Cr}$ ), alanine aminotransferase (ALT) and aspartate aminotransferase (AST) measured. If drug eruptions/abnormal laboratory tests developed during monitoring, allopurinol was stopped. The electronic dispensing system and computerized medical records were used for collection of patient demographics, indication for allopurinol use, initiation dose, monitoring intervals, laboratory results and clinical features of drug allergy. This was compared with the control group without an SMP. Chi square tests were used to compare differences in proportions and Mann-Whitney $U$ test for differences in medians. $P$ value $\leq 0.05$ was considered statistically significant.

Results: There were 61 cases and 30 controls with comparable age $(p=0.81)$, ethnicity ( $>80 \%$ Chinese) $(p=0.63)$ and estimated glomerular filtration rate, eGFR $(p=0.72)$. There were significantly more cases with tophaceous gout $(41 \%$ vs $10 \%, p=0.003)$, while more controls tumour lysis syndrome prophylaxis $(30 \%$ vs $0 \%, p<0.001$ ). Median (interquartile range, IQR) starting dose of 50 (50) mg was lower among cases versus controls of $100(200) \mathrm{mg}(\mathrm{p}<0.001)$; all cases had baseline and follow-up laboratory tests compared to controls $(p<0.001)$. Cases were followed up at a median (IQR) of 2 (1.1) weeks after initiation then 5 (2.0) weeks after the first visit, whereas controls were reviewed 8 (8.9) weeks after initiation, then 11 (4.6) weeks after the first visit. Two patients in the SMP group with normal eGFR developed maculopapular eruption (MPE), 1 elevated ALT/AST, and 1 both MPE and elevated ALT/AST within the first 14 days of initiation. One control with lymphoma and baseline eGFR $31 \mathrm{ml} / \mathrm{min} / 1.73 \mathrm{~m}^{2}$ developed DiHS (fever, MPE, elevated ALT/AST less than twice upper limit of normal) 43 days after initiation for tumour lysis prophylaxis. This occurred while on 2-weekly monitoring of FBC, ALT, AST. There were no cases of SCAR in both groups. 